\title{
Estresores académicos percibidos por estudiantes universitarios de ciencias de la salud
}

\author{
Academic stressors perceived by University studies of health sciences
}

\author{
R. González Cabanach, R. Fernández Cervantes, L. González Doniz, C. Freire \\ Rodríguez
}

Universidad de A Coruña, A Coruña, España

\begin{abstract}
Resumen
El propósito de esta investigación es identificar qué situaciones del contexto académico son percibidas como generadoras de estrés en una muestra de estudiantes de ciencias de la salud y en qué medida lo hacen. Adicionalmente, se analizan las diferencias en estos estresores académicos percibidos en función de las variables contextuales de la titulación y el curso. Para la medida de los estresores académicos ha sido empleada la escala de estresores académicos, que forma parte del cuestionario de estrés académico. Los resultados de este trabajo revelan que los exámenes y las intervenciones en público, por este orden, son las 2 situaciones académicas percibidas como más estresantes. Estos resultados sugieren que las situaciones más directas y claramente relacionadas con la evaluación son las que generan un mayor estrés a los estudiantes. Muy próximos se encuentran también los promedios resultantes en los factores sobrecarga del estudiante y deficiencias metodológicas del profesorado, factores que podemos considerar comparten la característica de externalidad e incontrolabilidad con respecto a los anteriores. Asimismo, existen diferencias significativas entre los grupos de estudiantes establecidos en función de la titulación que cursan en los estresores sobrecarga del estudiante, carencia de valor de los contenidos de aprendizaje y estudio y baja autoestima académica. Por lo que respecta a la variable curso, nuestros datos indican que existen diferencias significativas en los estresores falta de control sobre el propio rendimiento, exámenes e imposibilidad de participar en las decisiones respecto del propio trabajo académico, siendo el grupo de segundo el que obtiene la puntuación media más elevada en los 3 casos.
\end{abstract}

\begin{abstract}
This study aimed to identify those situations within the academic context that are perceived as stress generators in a sample of university students of Health Sciences and the amount of their impact. We also analyzed the differences in the academic stressors perceived based on the variables of career and academic year. These were measured with the Academic Stress Questionnaire, which forms a part of the Academic Stress Questionnaire (ASQ). The results of this project show that Exams and Public Participation are, in this order, the academic stressors most perceived. These findings suggest that the situations that are most directly and clearly related with the evaluation are those that generate the most stress to the students. The result averages in the factors Student burden and Teacher methodological shortcomings are very close to the above findings. We can consider that these four factors share the fact that they are external and uncontrollable in regards to the previous ones. In addition, there are significant differences among students groups of students established based on the career they are studying in the stressors Student burden, Low valuation of study and learning contents and Low academic self-concept. Regarding the academic year factor, our results show that there are significant differences in the following 3 stressors: Lack of control about own academic achievement, Exams and Impossibility of participating in decisions about one's own academic work. In these cases, second year students show, on average, the highest scores.
\end{abstract}

Palabras clave

Estresores académicos; Evaluación; Aprendizaje

Keywords

Academic stressors; Evaluation; Learning 


\section{Introducción}

Doménech $^{1}$ destaca la atención creciente que en la última década se le está dedicando al tema del estrés y del burnout del profesor. Este interés se ha traducido en una importante actividad investigadora, como queda reflejado en los numerosos trabajos publicados al respecto ${ }^{2,3,4}$ and 5 . Por una parte, los investigadores han tratado de identificar cuáles son los estresores más importantes que afectan al profesorado y que se han asociado con altos niveles de burnout, depresión y absentismo. Por otra parte, han centrado su atención en analizar el papel moderador que podrían tener algunas variables personales y sociales relevantes sobre el estrés de este grupo profesional.

El notable desarrollo de la investigación sobre el estrés del profesor contrasta con la escasa atención dedicada al estudio del estrés del estudiante. Con la salvedad de los trabajos que se ocupan de la ansiedad ante los exámenes y el estrés académico relacionado con la evaluación, cabe señalar que una revisión de la literatura indica que esta población ha carecido de una atención prioritaria en la investigación sobre estrés $^{6}$. No obstante, existe un pequeño número de estudios que, tomando como referente modelos del ámbito organizacional, tratan de abordar esta problemática ${ }^{7 \text { and } 8}$. Además, el estudio del burnout o el síndrome de «estar quemado» en el trabajo también se ha ampliado a muestras preocupacionales, como el denominado burnout académico ${ }^{9}$.

Existe acuerdo en que el estrés forma parte de la vida de los estudiantes y en que tiene potencialmente un efecto negativo sobre el aprendizaje y el rendimiento académico ${ }^{10}$ and 11 . En concreto, Lumley y Provenzano $^{12}$ afirman que el estrés puede afectar al funcionamiento académico del estudiante universitario, interfiriendo en comportamientos adaptativos tales como la dedicación al estudio y la asistencia a las clases o dificultando procesos cognitivos esenciales como son la atención y la concentración.

Cuando se trata de explicar el estrés académico, se pueden diferenciar 3 dimensiones: 1) Los estresores académicos o estímulos del ambiente educativo que son experimentados por el estudiante como una presión excesiva; 2) Las consecuencias del estrés académico sobre la salud y el bienestar psicológico del estudiante, su funcionamiento cognitivo y socioafectivo, su rendimiento académico, etc.; 3) Las variables moduladoras, o mediadores del estrés, entre las que se encuentran factores de naturaleza biológica (sexo, edad...), personal (apoyo social, rasgo de ansiedad, patrón de conducta tipo A, locus de control, autoeficacia, autoestima...), social (apoyo social, apoyo emocional...), psicoeducativa (tipo de estudios, curso...) y socioeconómica (lugar de residencia, disfrute de becas, nivel de ingresos familiares...). En este artículo nos centraremos en la primera de estas dimensiones: los estresores académicos.

Además de los problemas de transición y adaptación al nuevo escenario educativo ${ }^{13}$ y de $\operatorname{los}_{\text {factores }}$ relativos a las condiciones de la organización, el estudio de las dimensiones ligadas al proceso de enseñanza y aprendizaje es el que adquiere mayor relevancia en el abordaje del estrés en contextos académicos.

Diversas investigaciones han identificado la evaluación como la influencia contextual más crítica ${ }^{14}$ y generadora de estrés académico. En este sentido, se ha señalado que las pruebas y los exámenes a las que habitualmente tienen que enfrentarse los estudiantes suponen un tipo crítico de situaciones de estrés ${ }^{15}$.

Parecen bastante lógicas estas conclusiones por distintas razones: el temor a la evaluación, el miedo al fracaso, factores de índole cognitiva y afectiva y el constituir una condición básica para acceder a otras opciones formativas $\mathrm{u}$ ocupacionales futuras son algunos de los motivos más habitualmente contemplados. Además, la evaluación afecta notablemente a las creencias motivacionales (creencias de autoeficacia, creencias de capacidad, creencias de control...) y a la satisfacción y el bienestar psicológico del estudiante.

A estas razones señaladas cabe añadir la sobrecarga (cuantitativa y cualitativa) y la ambigüedad de rol que, con frecuencia, implica la evaluación académica: la concentración de exámenes en ciertos periodos del curso; el alto nivel de exigencia o la gran cantidad de materia que pueden abarcar; la incertidumbre acerca de las expectativas del profesor, de cómo va a valorar y calificar este el rendimiento y el aprendizaje y, en consecuencia, de cuál es el mejor modo de preparar el examen; las dudas sobre la adecuación de la forma de evaluación a lo impartido por el profesor y a los objetivos de la asignatura, etc.

Dentro de los estresores relacionados con el proceso de enseñanza-aprendizaje, se ha concedido una especial relevancia al papel que desempeñan las relaciones sociales que se establecen entre los individuos que forman parte del grupo y especialmente a las de los estudiantes entre sí. El profesor define las expectativas y demandas que configuran los roles académicos; los estudiantes establecen entre sí relaciones y forman grupos humanos, en parte condicionados por el estudio, en parte como fruto del contacto humano y buscan su desarrollo académico en el interior de las aulas.

Podría afirmarse con rotundidad que una parte significativa del éxito académico depende de la habilidad para utilizar los recursos personales y sociales. Estas habilidades favorecen la obtención de un 
mayor provecho del proceso de aprendizaje, un mejor rendimiento con el mismo esfuerzo e incluso un mejor pronóstico de inserción laboral futura.

En diversos modelos explicativos del estrés, la participación en la toma de decisiones y el apoyo social de profesores y compañeros aparecen como los factores reductores de estrés más importantes, como consecuencia, entre otros aspectos, de su efecto sobre la satisfacción y el compromiso y la implicación en el estudio.

Un buen clima social favorece el desarrollo y perfeccionamiento de las habilidades de resolución de conflictos (tanto personales como sociales), especialmente las habilidades de negociación. Ello desempeña un papel de amortiguación o reducción del estrés. En efecto, el apoyo social contribuye a que el estudiante afronte más eficazmente la situación estresante o atenúa la experiencia de estrés cuando ya se ha producido ${ }^{16}$. Además, las relaciones sociales constituyen un importante recurso de afrontamiento ${ }^{17}$.

Se ha puesto de manifiesto también que la falta de participación es un consistente predictor del estrés. Esta falta de participación aparece correlacionada, además, con diversos factores de riesgo para la salud mental y física: ánimo deprimido, baja autoestima, escasa satisfacción y motivación en el estudio. Asimismo, la falta de autonomía en el trabajo académico, como consecuencia de una estrecha supervisión, se asocia con la aparición de estrés. Ello como resultado, principalmente, de la falta de control que el estudiante experimenta en su actividad diaria.

Esta falta de participación y de autonomía o margen de decisión del estudiante sobre su trabajo académico está muy relacionada con las creencias de control. La percepción de falta de control sobre su trabajo (planificación, temporalización, elección de actividades, elección de estrategias...) y las escasas posibilidades de uso y desarrollo de sus capacidades personales en el trabajo académico favorecen la aparición de estrés.

Pero probablemente uno de los factores más claramente ligados a la aparición de estrés en el contexto académico sea la sobrecarga de trabajo. Dentro de esta sobrecarga hay que diferenciar entre el exceso de trabajo (sobrecarga cuantitativa) y la excesiva dificultad y complejidad del mismo (sobrecarga cualitativa). Como consecuencia de la limitación del tiempo disponible para dedicarlo a sus estudios, de sus propias limitaciones o como resultado de un plan de estudios con un número excesivo de asignaturas o con un grado de exigencias excesivamente elevado, entre otras razones, el estudiante se ve sobrepasado en sus recursos.

El objetivo de esta investigación es doble: por un lado, identificar qué situaciones del contexto académico relacionadas con el proceso de enseñanza y aprendizaje son percibidas como generadoras de estrés por una muestra de estudiantes de ciencias de la salud y en qué medida lo hacen. Por otro, estudiar las diferencias en estos estresores académicos percibidos en función de 2 variables contextuales relevantes: la titulación y el curso.

\section{Método}

Sujetos

La muestra de individuos utilizada la integran estudiantes de diversas titulaciones universitarias vinculadas a las ciencias de la salud de la Universidad de A Coruña. Los sujetos han sido seleccionados a partir de un muestreo por conglomerados, para respetar el agrupamiento natural de los estudiantes, considerando por ello, como conglomerado cada grupo clase integrado por aquellos estudiantes que voluntariamente colaboraron en la cumplimentación de los cuestionarios (muestra aceptante).

De este modo, la muestra está compuesta por un total de 258 estudiantes, 228 mujeres y 30 hombres, con edades comprendidas entre los 18-45 años, siendo la edad media de 21 años. Del total de la muestra, 131 sujetos $(50,8 \%)$ cursaban fisioterapia, 86 sujetos $(33,3 \%)$ estudiaban enfermería y 41 sujetos $(15,9 \%)$ eran alumnos de podología. En cuanto a la distribución de la muestra por cursos, 74 de los estudiantes participantes en el estudio cursaban primero, 110 estudiaban segundo y 84 tercero.

\section{Procedimiento}

Los datos relativos a las variables estudiadas fueron recogidos en cada uno de los centros a los que asistían los estudiantes que participaron en la investigación. Los cuestionarios fueron aplicados en un único momento temporal por personal especializado que colaboró en el estudio. A los participantes, que respondieron de forma individual y sin límite de tiempo a cada uno de los ítems del cuestionario, se les 
recalcó que era muy importante que respondieran con sinceridad a todas las cuestiones planteadas. Asimismo, se les informó del carácter totalmente confidencial de dicha información.

\section{Instrumentos de medida}

Para medir los estresores académicos, hemos empleado la escala de estresores académicos, que forma parte del cuestionario de estrés académico ${ }^{18}$. Esta escala de estimación está compuesta por un total de 54 ítems que pretenden medir diferentes situaciones y/o circunstancias del contexto académico que pueden presionar de algún modo al estudiante de manera que este los valore como un peligro o una amenaza real para su bienestar. Las respuestas a cada uno de los ítems se realizaron sobre una escala de 5 puntos desde «nunca» (1) hasta «siempre» (5).

Para evaluar el grado en que esta escala media constructos multidimensionales sometimos los datos a un análisis factorial exploratorio usando como método de extracción el análisis de componentes principales y como método de rotación la normalización Varimax con Kaiser. La medida de adecuación muestral de Kaiser-Meyer-Olkin, que es de 0,952, y la prueba de esfericidad de Bartlett, cuyo nivel de significación resultante es de 0,000, respaldan lo apropiado de esta factorización de las variables.

El estudio de la dimensionalidad de esta escala muestra una estructura factorial de 9 dimensiones (tabla 1), que logran explicar el 67,46\% de la varianza total. El primer factor está constituido por 12 ítems referidos a aspectos deficitarios en el proceso de enseñanza desarrollado por los profesores y que hemos denominado «deficiencias metodológicas del profesorado». Dentro del segundo factor, al que nos referiremos como «sobrecarga del estudiante», se agrupan 10 ítems relacionados con la percepción de que las demandas académicas resultan excesivas y con la escasez de tiempo para abordarlas. En el tercer factor se integran 5 ítems relacionados con todo tipo de intervenciones ante los demás (salir a la pizarra, hablar en voz alta en clase, realizar una exposición...), por lo que lo denominamos «intervenciones en público».

Tabla 1. Dimensiones de la escala estresores académicos del cuestionario de estrés académico (C.E.A.)

\begin{tabular}{|c|c|c|c|}
\hline Factor & Ítems & Ejemplo & Fiabilidad \\
\hline $\begin{array}{l}\text { I: Deficiencias metodológica } \\
\text { del profesorado }\end{array}$ & $\begin{array}{l}10,11,12,13,14 \\
15,16,17,18,19 \\
20,21\end{array}$ & $\begin{array}{l}\text { «Me pongo nervioso o me inquieto cuando los profesores no se ponen } \\
\text { de acuerdo entre ellos (manifiestan claras discrepancias entre ellos en } \\
\text { temas académicos)» }\end{array}$ & 0,9315 \\
\hline II: Sobrecarga del estudiante & $\begin{array}{l}27,29,31,32,33 \\
34,36,38,39,40\end{array}$ & $\begin{array}{l}\text { «Me pongo nervioso o me inquieto por las demandas excesivas y } \\
\text { variadas que se me hacen» }\end{array}$ & 0,9197 \\
\hline III: Intervenciones en público & $1,2,3,4,9$ & «Me pongo nervioso o me inquieto cuando me preguntan en clase» & 0,9240 \\
\hline IV: Clima social negativo & $\begin{array}{l}49,50,51,52,53 \\
54\end{array}$ & $\begin{array}{l}\text { «Me pongo nervioso o me siento inquieto por la ausencia de un buen } \\
\text { ambiente en clase» }\end{array}$ & 0,8463 \\
\hline $\begin{array}{l}\text { V: Falta de control sobre el } \\
\text { propio rendimiento } \\
\text { académico }\end{array}$ & $\begin{array}{l}24,28,30,43,44 \\
46\end{array}$ & «No sé qué hacer para que se reconozca mi esfuerzo y trabajo» & 0,8923 \\
\hline $\begin{array}{l}\text { VI: Carencia de valor de los } \\
\text { contenidos }\end{array}$ & $22,23,24,25$ & «Me preocupa que las asignaturas que cursamos tienen escaso interés» & 0,8610 \\
\hline $\begin{array}{l}\text { VII: Baja autoestima } \\
\text { académica }\end{array}$ & $26,35,37,41,42$ & $\begin{array}{l}\text { «Me pongo nervioso o me siento inquieto porque no creo que pueda } \\
\text { lograr los objetivos propuestos» }\end{array}$ & 0,8819 \\
\hline VIII: Exámenes & $5,6,7,8$ & «Me pongo nervioso o me inquieto mientras preparo los exámenes» & 0,8879 \\
\hline $\begin{array}{l}\text { IX: Falta de participación en } \\
\text { el propio trabajo Académico }\end{array}$ & $45,47,48$ & $\begin{array}{l}\text { «Me pongo nervioso o me siento inquieto porque no tengo posibilidad } \\
\text { alguna o muy escasa de dar mi opinión sobre la metodología de } \\
\text { enseñanza de las materias del plan de estudios» }\end{array}$ & 0,7977 \\
\hline Total de la escala & & & 0,9612 \\
\hline
\end{tabular}


El cuarto factor reúne un total de 6 ítems relacionados con un ambiente social percibido como desfavorable para el estudiante. Le hemos denominado como «clima social negativo». Un quinto factor, constituido por 5 ítems, permitiría poner de manifiesto la percepción de «falta de control sobre el propio rendimiento académico» por parte del estudiante. El sexto factor, denominado «carencia de valor de los contenidos», reúne un total de 4 ítems en los que el estudiante valora el grado de preocupación que le produce que aquello que está estudiando carezca de interés, utilidad futura, valor práctico, etc.

Dentro del séptimo factor se agrupan una serie de ítems en los que el estudiante manifiesta sentirse poco competente para hacer frente a las demandas académicas. Por consiguiente, se consideró adecuado referirse al mismo en términos de «baja autoestima académica» El octavo factor está constituido por 4 ítems que hacen referencia a los «exámenes» Finalmente, el noveno factor está formado por 3 ítems referidos a la «falta de participación en el propio trabajo académico» (tabla 1).

Como se puede apreciar en la citada tabla, el coeficiente de fiabilidad para el total de la escala, medido con el alfa de Cronbach, es de $\alpha=0,96$, oscilando entre 0,79-0,85 los coeficientes de las diversas dimensiones medidas.

\section{Técnicas de análisis de datos}

Para el estudio del primero de nuestros objetivos, hemos recurrido a la obtención de los estadísticos descriptivos de la muestra (media y desviación típica).

Para el estudio del segundo de los objetivos, hemos realizado sucesivos ANOVA. Con ellos se trataba de determinar si existían diferencias significativas en la percepción de estresores académicos. Una vez rechazada la hipótesis de igualdad de medias entre los grupos establecidos, nos interesa conocer dónde en concreto se encuentran tales diferencias. Con este propósito recurrimos a un tipo particular de contrastes denominados comparaciones múltiples post hoc o comparaciones a posteriori (Scheffé o Games Howell). La decisión sobre el procedimiento o prueba más adecuada nos exige determinar previamente si es posible o no asumir varianzas iguales mediante el uso del estadístico de Levene, el cual permite contrastar la hipótesis de igualdad de varianzas.

\section{Resultados}

En general, puede afirmarse que los estudiantes de ciencias de la salud sólo perciben como estresantes una parte de las dimensiones del proceso de enseñanza y aprendizaje. Las puntuaciones obtenidas en otra parte de las dimensiones medidas indican su bajo efecto estresor (tabla 2). 
Tabla 2. Análisis descriptivos para la escala de estresores académicos

\begin{tabular}{|c|c|c|c|c|c|c|c|c|c|c|c|}
\hline \multirow{2}{*}{$\begin{array}{l}\text { Estadísticos } \\
\text { descriptivos }\end{array}$} & \multirow{2}{*}{$\begin{array}{c}\text { N. }{ }^{\circ} \\
\text { Estadístico }\end{array}$} & \multirow{2}{*}{$\begin{array}{c}\text { Mínimo } \\
\text { Estadístico }\end{array}$} & \multirow{2}{*}{$\begin{array}{c}\text { Máximo } \\
\text { Estadístico }\end{array}$} & \multicolumn{2}{|c|}{ M } & \multirow{2}{*}{$\begin{array}{c}\text { DT } \\
\text { Estadístico }\end{array}$} & \multirow{2}{*}{$\begin{array}{c}\text { Varianza } \\
\text { Estadístico }\end{array}$} & \multicolumn{2}{|c|}{ Asimetría } & \multicolumn{2}{|c|}{ Curtosis } \\
\hline & & & & Estadístico & Error tipo & & & Estadístico & Error tipo & Estadístico & Error tipo \\
\hline Exámenes & 258 & 1,00 & 5,00 & 3,4758 & 0,0602 & 0,96618 & 0,934 & 0,208 & 0,152 & 0,924 & 0,302 \\
\hline $\begin{array}{l}\text { Intervenciones en } \\
\text { público }\end{array}$ & 258 & 1,00 & 5,00 & 3,3116 & 0,0698 & 1,12042 & 1,255 & 0,030 & 0,152 & $-1,215$ & 0,302 \\
\hline $\begin{array}{l}\text { Sobrecarga del } \\
\text { estudiante }\end{array}$ & 258 & 1,00 & 5,00 & 2,9388 & 0,544 & 0,87448 & 0,765 & 0,183 & 0,152 & $-0,854$ & 0,302 \\
\hline $\begin{array}{l}\text { Deficiencia } \\
\text { metodológica del } \\
\text { profesorado }\end{array}$ & 258 & 1,17 & 5,00 & 2,9351 & 0,0537 & 0,86219 & 0,743 & 0,265 & 0,152 & $-0,641$ & 0,302 \\
\hline $\begin{array}{l}\text { Inseguridad respecto } \\
\text { del propio } \\
\text { rendimiento }\end{array}$ & 258 & 1,00 & 5,00 & 2,5163 & 0,0567 & 0,91088 & 0,830 & 0,584 & 0,152 & $-0,231$ & 0,302 \\
\hline $\begin{array}{l}\text { Falta de control sobre } \\
\text { el propio rendimiento }\end{array}$ & 258 & 1,00 & 4,80 & 2,2481 & 0,0537 & 0,86214 & 0,743 & 0,613 & 0,152 & $-0,178$ & 0,302 \\
\hline $\begin{array}{l}\text { Malas relaciones } \\
\text { sociales en el contexto } \\
\text { académico }\end{array}$ & 258 & 1,00 & 5,00 & 2,2681 & 0,0549 & 0,88127 & 0,777 & 0,665 & 0,152 & $-0,204$ & 0,302 \\
\hline $\begin{array}{l}\text { Imposibilidad de } \\
\text { participar en las } \\
\text { decisiones respecto } \\
\text { del propio trabajo } \\
\text { académico }\end{array}$ & 258 & 1,00 & 5,00 & 2,2313 & 0,0557 & 0,89453 & 0,800 & 0,894 & 0,152 & 0,437 & 0,302 \\
\hline $\begin{array}{l}\text { Carencia de valor de } \\
\text { los contenidos de } \\
\text { aprendizaje y estudio }\end{array}$ & 258 & 1,00 & 5,00 & 2,1880 & 0,0561 & 0,90073 & 0,811 & 0,907 & 0,152 & 0,369 & 0,302 \\
\hline $\begin{array}{l}\text { N. }{ }^{\circ} \text { válido (según } \\
\text { lista) }\end{array}$ & 258 & & & & & & & & & & \\
\hline
\end{tabular}

DT: desviación típica; M: mediana. 
Como se puede observar en la tabla 2, los exámenes y las intervenciones en público, por este orden, son las 2 situaciones académicas que estos estudiantes perciben como más estresantes, situándose en ambos casos las puntuaciones medias por encima del valor «3». Muy próximos a este valor se encuentran también los promedios de los factores sobrecarga del estudiante y deficiencias metodológicas del profesorado, de 2,93 en ambos casos. Por lo que se refiere a las circunstancias que los universitarios de nuestra muestra valoran como menos amenazantes para su bienestar en contextos académicos, nos encontramos con que es el factor carencia de valor de los contenidos de aprendizaje y estudio el que obtiene la puntuación media más baja $(M=2,18)$. En el punto inferior de la tabla se encuentra también la falta de participación (M=2,23) (tabla 3).

Tabla 3. Medias, desviaciones típicas y significación estadística de las diferencias entre estudiantes de las 3 titulaciones consideradas en el estudio respecto a la percepción de estresores académicos

\begin{tabular}{|c|c|c|c|c|c|}
\hline & $\mathrm{N} .^{\circ}$ & M & DT & $\mathrm{F}$ & Sig. \\
\hline \multicolumn{6}{|c|}{ Deficiencias metodológicas del profesorado } \\
\hline Enfermería & 86 & $\mathbf{3 , 0 3}$ & 0,87 & 1,693 & 0,186 \\
\hline Podología & 41 & 2,73 & 0,87 & & \\
\hline Fisioterapia & 131 & 2,93 & 0,84 & & \\
\hline \multicolumn{6}{|c|}{ Sobrecarga del estudiante } \\
\hline Enfermería & 86 & 2,75 & 0,72 & 16,601 & $\mathbf{0 , 0 0 0}$ \\
\hline Podología & 41 & 2,44 & 0,72 & & \\
\hline Fisioterapia & 131 & 3,21 & 0,91 & & \\
\hline \multicolumn{6}{|c|}{ Intervenciones en público } \\
\hline Enfermería & 86 & 3,42 & 1,12 & 2,497 & 0,084 \\
\hline Podología & 41 & 2,96 & 1,15 & & \\
\hline Fisioterapia & 131 & 3,34 & 1,09 & & \\
\hline \multicolumn{6}{|c|}{ Clima social negativo } \\
\hline Enfermería & 86 & 2,21 & 0,86 & 1,144 & 0,320 \\
\hline Podología & 41 & 2,13 & 0,90 & & \\
\hline Fisioterapia & 131 & 2,34 & 0,88 & & \\
\hline \multicolumn{6}{|c|}{ Falta de control sobre el propio rendimiento } \\
\hline Enfermería & 86 & 2,34 & 0,84 & 1,796 & 0,168 \\
\hline Podología & 41 & 2,12 & 0,74 & & \\
\hline Fisioterapia & 131 & 2,41 & 0,90 & & \\
\hline \multicolumn{6}{|c|}{ Carencia de valor de los contenidos de aprendizaje y estudio } \\
\hline Enfermería & 86 & 2,37 & 0,93 & 4,452 & $\mathbf{0 , 0 1 3}$ \\
\hline Podología & 41 & 2,31 & 0,83 & & \\
\hline Fisioterapia & 131 & 2,02 & 0,87 & & \\
\hline \multicolumn{6}{|c|}{ Baja autoestima académica } \\
\hline Enfermería & 86 & 2,37 & 0,80 & 4,344 & 0,014 \\
\hline Podología & 41 & 2,29 & 0,82 & & \\
\hline Fisioterapia & 131 & 2,67 & 0,97 & & \\
\hline \multicolumn{6}{|l|}{ Exámenes } \\
\hline Enfermería & 86 & 3,56 & 0,93 & 0,866 & 0,422 \\
\hline Podología & 41 & 3,32 & 1,01 & & \\
\hline Fisioterapia & 131 & 3,46 & 0,97 & & \\
\hline \multicolumn{6}{|c|}{ Falta de participación En el propio trabajo académico } \\
\hline Enfermería & 86 & 2,35 & 0,92 & 1,608 & 0,202 \\
\hline Podología & 41 & 2,26 & 0,94 & & \\
\hline Fisioterapia & 131 & 2,13 & 0,85 & & \\
\hline
\end{tabular}

DT: desviación típica; M: mediana; Sig.: significación estadística. 
Como podemos observar en la tabla 3, existen diferencias significativas entre los grupos de estudiantes en función de la titulación que cursan en la percepción de los siguientes estresores: sobrecarga del estudiante, carencia de valor de los contenidos de aprendizaje y estudio y baja autoestima académica. Todas estas diferencias resultan significativas (al nivel de $\mathrm{p}<0,001$, en el primer caso y al nivel de $\mathrm{p}<0,05$, en $\operatorname{los} 2$ restantes).

Tanto la percepción de sobrecarga del estudiante como la baja autoestima académica como estresores académicos se dan en mayor medida entre los estudiantes de fisioterapia, en comparación con los estudiantes de enfermería y podología, no alcanzando significación estadística la diferencia entre estos últimos.

En cuanto a la carencia de valor de los contenidos de aprendizaje y estudio, los contrastes post hoc realizados nos indican que son los estudiantes de enfermería los que valoran como más estresante esta variable, diferenciándose de manera significativa de los estudiantes de fisioterapia pero no de los de podología. Tampoco alcanzan significación estadística las diferencias entre estos 2 últimos grupos de estudiantes.

Por lo que respecta a la variable curso, los resultados expuestos en la tabla 4 señalan la existencia de diferencias significativas entre los grupos de estudiantes de los distintos cursos en la percepción de los estresores falta de control sobre el propio rendimiento, exámenes e imposibilidad de participar en las decisiones respecto del propio trabajo académico (tabla 4). 
Tabla 4. Medias, desviaciones típicas y significación estadística de las diferencias entre estudiantes de los 3 cursos considerados en el estudio respecto a la percepción de estresores académicos

\begin{tabular}{|c|c|c|c|c|c|}
\hline & $N .^{\circ}$ & $M$ & $D T$ & $F$ & Sig. \\
\hline \multicolumn{6}{|c|}{ Deficiencias metodológicas del profesorado } \\
\hline Primero & 74 & 2,77 & 0,88 & 2,438 & 0,089 \\
\hline Segundo & 100 & 3,06 & 0,83 & & \\
\hline Tercero & 84 & 2,91 & 0,85 & & \\
\hline \multicolumn{6}{|c|}{ Sobrecarga del estudiante } \\
\hline Primero & 74 & 2,81 & 0,83 & 2,128 & 0,121 \\
\hline Segundo & 100 & $\mathbf{3 , 0 7}$ & 0,92 & & \\
\hline Tercero & 84 & 2,88 & 0,84 & & \\
\hline \multicolumn{6}{|c|}{ Intervenciones en público } \\
\hline Primero & 74 & 3,34 & 1,12 & 0,783 & 0,458 \\
\hline Segundo & 100 & 3,20 & 1,12 & & \\
\hline Tercero & 84 & 3,40 & 1,11 & & \\
\hline \multicolumn{6}{|c|}{ Clima social negativo } \\
\hline Primero & 74 & 2,24 & 0,96 & 0,043 & 0,958 \\
\hline Segundo & 100 & 2,28 & 0,86 & & \\
\hline Tercero & 84 & 2,27 & 0,83 & & \\
\hline \multicolumn{6}{|c|}{ Falta de control sobre el propio rendimiento } \\
\hline Primero & 74 & 2,27 & 0,89 & 0,3944 & 0,021 \\
\hline Segundo & 100 & 2,53 & 0,87 & & \\
\hline Tercero & 84 & 2,19 & 0,79 & & \\
\hline \multicolumn{6}{|c|}{ Carencia de valor de los contenidos de aprendizaje y estudio } \\
\hline Primero & 74 & 2,25 & 1,07 & 0,296 & 0,744 \\
\hline Segundo & 100 & 2,17 & 0,79 & & \\
\hline Tercero & 84 & 2,14 & 0,86 & & \\
\hline \multicolumn{6}{|c|}{ Baja autoestima académica } \\
\hline Primero & 74 & 2,55 & 1,08 & 0,403 & 0,669 \\
\hline Segundo & 100 & 2,55 & 0,85 & & \\
\hline Tercero & 84 & 2,44 & 0,80 & & \\
\hline \multicolumn{6}{|l|}{ Exámenes } \\
\hline Primero & 74 & 3,21 & 1,00 & $0,4,758$ & 0,009 \\
\hline Segundo & 100 & 3,67 & 0,92 & & \\
\hline Tercero & 84 & 3,47 & 0,93 & & \\
\hline \multicolumn{6}{|c|}{ Imposibilidad de participar en el propio trabajo académico } \\
\hline Primero & 74 & 2,03 & 0,83 & 6,428 & 0,002 \\
\hline Segundo & 100 & 2,47 & 0,95 & & \\
\hline Tercero & 84 & 2,11 & 0,81 & & \\
\hline
\end{tabular}

DT: desviación típica; M: mediana; Sig.: significación estadística.

Son los estudiantes de segundo curso los que perciben estas 3 dimensiones como estresantes, diferenciándose significativamente de los estudiantes de primero y tercero (en el caso de la baja autoestima académica), de los estudiantes de tercero (en el caso de la falta de control sobre el propio rendimiento) y de los estudiantes de primero (en el caso de los exámenes). 


\section{Conclusiones}

Los resultados de este trabajo nos permiten extraer una serie de conclusiones que analizaremos en relación con los objetivos de este trabajo. En primer lugar, podemos afirmar que son las situaciones más directa y claramente relacionadas con la evaluación, como son los exámenes y las intervenciones en público (cuando a uno le preguntan en clase, debe salir a la pizarra o hacer una exposición...) las que son percibidas como más estresantes por los estudiantes. Lo mismo puede afirmarse de la sobrecarga.

Ello coincide con los datos aportados por la literatura científica previa, que indicaba que la evaluación y las dimensiones relacionadas (sobrecarga, intervención en publico) son las que generan mayor estrés académico, $^{8,19}$ and 20.

También podemos afirmar que otra variable que genera estrés académico es la deficiencia metodológica del profesorado. Probablemente como consecuencia de su incontrolabilidad por el estudiante y de que el estilo de enseñanza marca las pautas de interacción entre el profesor y el alumno y define sus roles respectivos ${ }^{21}$.

En segundo lugar, y en relación con el segundo objetivo de nuestra investigación, nuestros resultados ponen de manifiesto que los estudiantes de fisioterapia se perciben más sobrecargados que los de enfermería y podología. Ello podría ser consecuencia de una mayor sobrecarga de tipo cualitativo, efecto de la mayor complejidad del trabajo académico desarrollado, ya que la carga total de créditos es mayor, en cambio, en las otras titulaciones.

En cambio, son los estudiantes de enfermería quienes perciben significativamente una mayor carencia de valor de los contenidos de aprendizaje y estudio. Es posible que el plan de estudios de enfermería no satisfaga las expectativas que los estudiantes de esta titulación tenían inicialmente con respecto a la misma, o bien que el rol profesional desempeñado les ponga en contacto con una realidad indicativa de carencias formativas.

Es en lo relativo a la variable curso donde nuestros resultados difieren claramente de los obtenidos en otros estudios previos que, por un lado, apuntan a los primeros cursos como los más vulnerables por encontrarse en un momento de transición y adaptación al sistema universitario ${ }^{13}$ o por no haber adquirido todavía adecuados mecanismos de afrontamiento eficaces de los estresores académicos ${ }^{22}$; o por otro lado, sugieren que el desencanto o no cumplimiento de expectativas que aparecen a lo largo de la socialización del estudiante en la universidad podría llevar a que los estudiantes de cursos superiores se mostrasen menos satisfechos con los estudios, la facultad y la universidad en general ${ }^{9}$.

Es posible que una explicación de estos resultados tenga que ver con el hecho de que actualmente en las titulaciones de ciencias de la salud la mayor carga práctica se centra en el segundo curso de la carrera, que es, a la vez, cuando comienzan las prácticas externas. Ello puede propiciar un incremento de la carga percibida por los estudiantes, que, además, han de enfrentarse a situaciones clínicas que no dominan y que, en algunos casos, representan una fuente importante de estrés. En cualquier caso, esta explicación no deja de ser una de las posibles, que habría de ser contrastada con estudios en otros ámbitos del conocimiento.

Estas conclusiones adquieren mayor relevancia en el actual momento de cambios en la enseñanza superior, aportando información para una más eficiente planificación de la enseñanza de grado en estas titulaciones y para una más adecuada orientación y apoyo a estos estudiantes en su desarrollo académico.

En cualquier caso, pensamos que debemos ser especialmente cautelosos a la hora de emitir juicios acerca de las diferencias encontradas tanto en función de la titulación como del curso en las diferentes dimensiones de estrés académico estudiadas, entre otras razones por la posibilidad de haber confundido los posibles efectos de la edad con los del curso, o incluso los del sexo con los del curso dada la falta de equilibrio entre hombres y mujeres en la configuración de la muestra. Nosotros entendemos que, para abordar el estudio de estas variables en la experiencia de estrés académico lo más apropiado sería, en el futuro, el empleo de un estudio de tipo longitudinal que nos permitiría ejercer el control sobre las variables de tipo personal.

\section{Conflicto de intereses}

Los autores declaran no tener ningún conflicto de intereses. 


\section{Bibliografía}

1. F. Doménech. Autoeficacia, recursos escolares de afrontamiento y agotamiento docente en profesores de secundaria. Infanc Aprendiz, 4 (2005), pp. 471-483.

2. E. Calvete, A. Villa. Estrés y Burnout docente: influencia de variables cognitivas. Rev Educación, 319 (1999), pp. 291-303.

3. D.W. Chan. Stress, self-efficacy, social support, and psychological distress among prospective Chinese teachers in Hong Kong. Educational Psychology, 22 (2002), pp. 557-569.

4. R. Van Dick, U. Wagner. Stress and strain in teaching: A structural equation approach. Br J Educ Psychol, 71 (2001), pp. 243-259.

5. M. Van Der Doef, S. Maes. Teacher-specific quality of work versus general quality of work assessment: A comparison of their validity regarding burnout, psychosomatic well-being and job satisfaction. Anxiety Stress Coping, 15 (2002), pp. 327-344.

6. F. Michie, M. Glachan, D. Bray. An evaluation of factors influencing the academic self-concept, self-esteem and academic stress for direct and re-entry students in Higher Education. Educational Psychology, 21 (2001), pp. 455-472.

7. M.J. Chambel, L. Curral. Stress in academic life: Work characteritics as predictors of student well-being and performance. J Appl Psychol, 54,1 (2005), pp. 135-147.

8. F.J. Muñoz. El estrés académico. Problemas y soluciones desde una perspectiva psicosocial. Servicio de Publicaciones de la Universidad de Huelva, Huelva (2004).

9. M. Salanova, I.M. Martínez, E. Bresó, S. Llorens, R. Grau. Bienestar psicológico en estudiantes universitarios: facilitadores y obstaculizadores del desempeño académico. Anal Psicología, 21, 1 (2005), pp. 170-180.

10. G. Felsten, K. Wilcox. Influences of stress, situation-specific mastery beliefs and satisfaction with social support on well-being and academic performance. Psychol Rep, 70 (1992), pp. 219-303.

11. C.W. Struthers, R.P. Perry, V.H. Menec. An examination of the relationships among academic stress, coping motivation and performance in college. Res High Educ, 41 (2000), pp. 581-592.

12. M.A. Lumley, K.M. Provenzano. Stress management through written emotional disclosure improves academic performance among college students with physical symptoms. J Educ Psychol, 95,3 (2003), pp. 641-649.

$\underline{13}$. E. Corominas, S. Isus. Transiciones y orientación. R.I.E, 16, 2 (1998), pp. 155-184.

14. D. Watkins, J. Hattie. A longitudinal study of the approaches to learning of Australian tertiary students. Human Learn, 4 (1985), pp. 127-141.

15. G. Splanger, R. Pekrun, K. Kramer, H. Hofmann. Students'emotions, physiological reactions, and coping in academic exams. Anxiety Stress Coping, 15,4 (2002), pp. 413-432.

16. B. Ojeda, J. Ramal, F. Calvo, R. Vallespín. Estrategias de afrontamiento al estrés y apoyo social. Psiquis, 22, 3 (2001), pp. 155-168.

17. A. Stevens, D. Coon, S. Wisniewski, D. Vance, S. Arguelles, S. Belle, et al. Measurement of leisure time satisfaction in family caregivers. Aging Ment Health, 8 (2004), pp. 450-459.

18. Cabanach RG, Valle A, Rodríguez S, Piñeiro, I. Respuesta de estrés en contextos universitarios: construcción de una escala de medida. Comunicación. V Congreso Internacional de Psicología y Educación: los retos de futuro. Oviedo (España) 23-25 de abril, 2008.

19. A. Escalona, J.J. Miguel Tobal. Ansiedad ante los exámenes: evolución histórica y aportaciones prácticas para su tratamiento. Ansiedad y Estrés, 2 (1996), pp. 195-209.

20. Magaz A, García EM, del Valle M. Ansiedad, estrés y problemas de ansiedad en escolares de 12 a 18 años. Pap Psicol. 1998, 71. Disponible en: http://www.papelesdelpsicologo.es/vernumero.asp?id=796.

21. J.I. Aragonés. El rol del maestro y del alumno. C (Comp). Huici (Ed.), Estructura y procesos de grupo, UNED, Madrid (1985), pp. 215-239.

22. A. Polo, J.M. Hernández, C. Pozo. Evaluación del estrés académico en estudiantes universitarios. Ansiedad y Estrés, 2 (1996), pp. 159-172. 